\section{Características de abortamentos atendidos em uma maternidade pública do Município da Serra - ES}

\section{Characteristics of hospital abortions in a public maternity in the municipality of Serra-ES}

\section{Resumo}

O objetivo do estudo foi descrever as características do abortamento de mulheres admitidas em uma maternidade pública do Espírito Santo. É um estudo transversal. A amostra constituiu-se de 21 mulheres que induziram o abortamento e 62 que o declararam como espontâneo, internadas no período de agosto de 2005 a janeiro de 2006. Para a coleta de dados, foi utilizado um formulário. Os dados foram analisados pelo SPSS, versão 14.0. Em ambos os tipos de abortamento, a maioria das mulheres $(85,5 \%)$ não planejou a gestação e a idade gestacional predominante foi de dez semanas e menos. Um maior percentual de mulheres do grupo do abortamento induzido foi aconselhado a abortar $(47,6 \%)$, apresentou hemorragia $(28,6 \%)$ e sinais de infecção $(19,1 \%)$ à admissão, e necessitou utilizar antibioticoterapia (19,1\%). O método de escolha para a prática do abortamento foi o Misoprostol (76,2\%). O pai do concepto teve maior participação na decisão do aborto do que a família. Os principais motivos que levaram à indução do abortamento foram falta de condições financeiras $(29,4 \%)$, falta de apoio do pai do concepto (20,6\%), e ter uma relação conjugal instável $(17,7 \%)$. Concluise que é necessário aumentar as possibilidades de se planejar a gestação e valorizar o Planejamento Familiar como um componente indispensável para o processo global de desenvolvimento social e econômico do País.

Palavras-chave: Abortamento. Saúde reprodutiva. Planejamento familiar.

\section{Priscilla Rocha Araújo Nader ${ }^{1}$}

\section{Vanez da Rocha Panetto Blandino²}

\section{Ethel Leonor Nóia Maciel ${ }^{3}$}

${ }^{1}$ Escola Superior de Ciências da Santa Casa de Misericórdia.

${ }^{2}$ Acadêmica de Enfermagem do Centro Superior de Ensino - UNIVIX.

${ }^{3}$ Departamento de Saúde Coletiva e Núcleo e Doenças Infecciosas, Universidade Federal do Espírito Santo.

Correspondência: Priscilla Rocha Araújo Nader, Rua Amélia Tartuce Nasser 835 apto. 404, Jardim da Penha, Vitória, ES, CEP 29060-110

E-mail: priscillarocha10@hotmail.com 
Abstract

The study aimed to describe the characteristics of abortion of women in a public hospital in the state of Espírito Santo. It is a cross-sectional study. The sample consisted of 21 women who had induced abortion, and 62 who declared miscarriage, hospitalized in the period between August 2005 and January 2006. A form was used for data collection. Data were analyzed by SPSS, version 14.0. In both kinds of abortion, most women $(85.5 \%)$ had not planned to become pregnant and the predominant gestational age was 10 weeks and less. A greater percentage of women from the induced abortion group were advised to abort (47.6\%), had bleedings (28.6\%) and signs of infection (19.1\%) at admission and needed antibiotic therapy (19.1\%). The method chosen to abort was Misoprostol (76.2\%). The concepto's father was more participative in the decision of abortion than the family. The main reasons for induced abortion were the lack of good financial situation (29.4\%), lack of father support (20.6\%), and non-stable marital status (17.7\%). The authors conclude it is necessary to increase the possibility of planning pregnancy and valuing Family Planning as an indispensable component for the global process of the Country's social and economic development.

Keywords: Abortion. Reproductive health. Family planning.

\section{Introdução}

Segundo dados do Relatório sobre a Situação da População Mundial, Fundo de População das Nações Unidas, ocorrem por ano, no mundo, 529 mil mortes maternas ${ }^{1}$.

Atualmente, o óbito de mulheres por causas ligadas à gravidez, ao parto e ao puerpério é, em grande parte, prevenível. Por esse motivo, a mortalidade materna é um indicador de condições de vida da população e de qualidade da atenção à saúde da mulher, o que abrange determinantes, como acesso aos serviços de saúde, concentração de renda, desenvolvimento de políticas sociais e até mesmo percepção da mulher em relação ao seu próprio corpo e aos seus direitos como cidadã. As altas taxas de mortalidade materna revelam precariedade na assistência à saúde ${ }^{2}$.

Van Look e Cottingham ${ }^{3}$ expõem que a qualidade dos serviços de contracepção é um elemento essencial para a estratégia de redução do abortamento inseguro e, conseqüentemente, da mortalidade materna, $o$ que abrange acesso adequado às informações sobre métodos contraceptivos e conhecimento de como obter esses métodos.

O Código Penal Brasileiro institui o abortamento provocado como crime, sob pena de detenção ou reclusão; entretanto, o artigo 28 delibera que não se pune abortamento provocado por médico nos casos de aborto necessário, se não houver outro meio de salvar a vida da gestante, e também nos casos de gravidez resultante de estupro 4 .

No entanto, essas medidas legais não diminuem a prática do abortamento induzido no País e sim perpetuam as desigualdades socioeconômicas presentes na história do Brasil. Mulheres com recursos financeiros têm acesso a métodos seguros de interrupção da gestação, enquanto outras, de classes menos abastadas, são obrigadas a se submeter a procedimentos inseguros que podem até colocá-las em condições de risco de morte ${ }^{5}$.

O abortamento legal tem como conseqüência uma taxa de mortalidade de menos de um óbito por 100.000 nascidos vi- 
vos; já o abortamento clandestino possui uma taxa, significativamente maior, de 50 a 100 mortes por 100.000 nascidos vivos ${ }^{6}$.

Estima-se que de 40-50 milhões de abortamentos que ocorrem anualmente no mundo, cerca da metade deles ocorrem em condições inseguras. Globalmente, cerca de $13 \%$ de todas as mortes maternas devemse a complicações do abortamento ${ }^{7}$.

Monteiro e Adesse ${ }^{8}$, aceitando que $20 \%$ das mulheres que induziram um abortamento tiveram que ser internadas por suas complicações, encontraram que, no Brasil, de 1992 a 2005 houve diminuição nas internações por abortamento no SUS, passando de 344.956 internações em 1992 para 252.917 em 1996, mantendo-se valores próximos a 250.000 internações em 2005. Ao estimar o número de abortamentos induzidos, no País, encontraram a ocorrência de 1.054.242 em 2005.

Apesar de, não ser possível saber exatamente a dimensão dos casos de abortamento provocados devido a seu caráter ilegal e também à carência de indicadores que permitam quantificar o real número de episódios, sabe-se que, no contexto da mortalidade materna brasileira, a incidência de óbitos por complicações do abortamento oscila em torno de $12,5 \%$, ocupando o quarto lugar entre as causas observadas ${ }^{9}$.

Com o intuito de contribuir com o debate sobre o abortamento como problema de saúde pública, essa pesquisa teve por objetivo descrever as características do abortamento de mulheres admitidas em uma maternidade do município da Serra, Espírito Santo.

\section{Materiais e Métodos}

\section{Delineamento do estudo.}

Trata-se de um estudo descritivo de corte transversal.

\section{Coleta de Dados}

A pesquisa foi desenvolvida na Maternidade de Carapina, instituição pública da
Prefeitura Municipal da Serra - ES, que atende pela razão social de Maternidade e Pronto-Atendimento Coronel Leôncio Vieira de Rezende. 48,8\% dos partos realizados no referido município, na rede pública, são efetuados nessa maternidade ${ }^{10}$. Em um levantamento realizado no ano de 2004, das 1.618 internações, 204 se deveram a abortamento, o que representa $12,60 \%$ das internações, considerando a admissão de gestante para tratamento clínico, e 13,50\% no término das gestações ${ }^{11}$. A maternidade presta assistência a gestantes, parturientes, puérperas e recém-nascidos de baixo risco, a maioria residente no referido município. Foram selecionadas para participar do estudo mulheres admitidas com diagnóstico de abortamento, no período de agosto de 2005 a janeiro de 2006.

Foi construído, para levantamento das variáveis, um formulário contendo perguntas abertas e fechadas. Este instrumento foi subdividido em 3 seções: características sociodemográficas; características reprodutivas e características do abortamento. Foi realizado um estudo piloto para validação do instrumento no mês de julho de 2005. A coleta das informações e o registro no instrumento foram realizados pelas próprias pesquisadoras. A definição do tipo de abortamento (espontâneo ou induzido) foi obtida através dos critérios estabelecidos pela $\mathrm{OMS}^{12}$. Foram considerados abortamentos induzidos aqueles que se encaixaram na categoria "aborto seguramente provocado", identificados a partir da declaração da mulher. Nos casos que tiveram indicação de curetagem, tomou-se o cuidado de realizar as entrevistas sempre após o procedimento, levando em conta que antes da referida intervenção a mulher encontra-se, de maneira geral, sangrando, com dor e ansiosa, o que poderia acarretar desconforto por parte da entrevistada.

Durante o período estudado foram internadas 108 mulheres com diagnóstico de abortamento. Pelo fato de duas das autoras trabalharem na Maternidade de Carapina, não houve dificuldade para se abordar o máximo de casos possível, não ocorreram 
recusas e as perdas se deveram a dificuldade de acesso das autoras às mulheres no intervalo de tempo entre a curetagem e a alta hospitalar.

\section{Calculo de tamanho da amostra}

Utilizando os procedimentos de tamanho de amostra para uma proporção em uma população, considerando o tamanho da população como infinita (grande), precisão desejada de $80 \%$, incidência de aborto induzido de $20 \%$ e nível de significância de $5 \%$, determinamos o tamanho da amostra em 61 mulheres. Definimos o plano amostral elegendo os cinco dias úteis da semana para realizar as entrevistas.

\section{Análise dos dados}

Como métodos de análise descritiva, foi empregada distribuição de freqüência absoluta e relativa para variáveis qualitativas. Para a análise dos dados, os abortamentos foram estratificados em espontâneos e induzidos, o teste qui-quadrado foi utilizado com um intervalo de confiança de 95\%, e os dados foram analisados no programa Social Package Statistical Science (SPSS) versão 14.0 (2006).

\section{Considerações éticas}

As pacientes foram abordadas na enfermaria, o objetivo do estudo foi explicado e só participaram do experimento se, após informação sobre a pesquisa, apresentaram o desejo de contribuir com o estudo, assinando um Termo de Consentimento Livre e Esclarecido.

Este projeto foi aprovado pelo Comitê de Ética em Pesquisa da Universidade Federal do Espírito Santo, sob o número 025/ 05, e autorizado pela direção da Maternidade de Carapina.

\section{Resultados}

Entre os 83 casos de abortamento estudados, durante o período de agosto de
2005 a janeiro de 2006, 21 mulheres admitiram ter provocado abortamento, o que corresponde a $25,3 \%$ do total.

A maior parte das mulheres, dos dois grupos, não planejou a gestação (Tabela 1). Houve significância estatística $(p=0,029)$ na comparação entre as diferenças dos grupos, pois, como esperado, todas as mulheres do grupo do abortamento induzido referiram não ter planejado a gestação, contra $80,6 \%(n=50)$ do grupo do abortamento espontâneo.

Em relação ao desejo da gestação, entre as mulheres do grupo do abortamento espontâneo que responderam a essa pergunta, 76,5\% (n=39) referiram ter desejado a gravidez. É interessante citar que quatro entrevistadas pertencentes ao grupo do abortamento induzido referiram ter desejado a gestação, apesar de não a terem levado até o fim. A diferença entre os dois grupos foi estatisticamente significante $(\mathrm{p}=0,000)$.

Em ambos os tipos de abortamentos, a idade gestacional predominante foi de dez semanas e menos, representando $48,4 \%$ $(n=30)$ dos abortamentos espontâneos e $52,4 \%(n=11)$ dos induzidos.

Um maior percentual de mulheres do grupo do abortamento induzido $(28,6 \%$, $\mathrm{n}=6$ ) apresentou hemorragia grave na admissão, em comparação com as entrevistadas do grupo do abortamento espontâneo $(16,1 \%, n=10)$. Quando perguntadas sobre sinais de infecção na admissão hospitalar, $8,1 \%(\mathrm{n}=5)$ das mulheres do grupo do abortamento espontâneo e 19,1\% $(n=4)$ das entrevistadas do grupo do abortamento induzido responderam afirmativamente. Das cinco mulheres que referiram ter apresentado sinais de infecção na admissão hospitalar, do grupo do abortamento espontâneo, apenas duas fizeram uso de antibiótico no decorrer da internação; em contrapartida, as quatro mulheres do grupo do abortamento induzido que referiram sinais de infecção utilizaram a medicação citada, sendo o valor de $\mathrm{p}=0,016$.

Observa-se, na Tabela 2, que apenas $17,7 \%(\mathrm{n}=11)$ das entrevistadas do grupo 
Tabela 1 - Características reprodutivas estratificadas de acordo com tipo de abortamento.

Table 1 - Reproductive characteristics stratified by type of abortion.

\begin{tabular}{|c|c|c|c|c|c|}
\hline \multirow[b]{2}{*}{ Características } & \multicolumn{2}{|c|}{ Aborto Espontâneo } & \multicolumn{2}{|c|}{ Aborto Induzido } & \multirow[t]{2}{*}{$p$} \\
\hline & $\mathrm{N}$ & $\%$ & $\mathrm{~N}$ & $\%$ & \\
\hline \multicolumn{6}{|c|}{ Gravidez planejada } \\
\hline Sim & 12 & 19,4 & 0 & 0 & 0,029 \\
\hline Não & 50 & 80,6 & 21 & 100 & \\
\hline Total & 62 & 100 & 21 & 100 & \\
\hline \multicolumn{6}{|c|}{ Gravidez desejada } \\
\hline Sim & 39 & 76,5 & 4 & 19,0 & 0,000 \\
\hline Não & 12 & 23,5 & 17 & 81,0 & \\
\hline Total & *51 & 100 & 21 & 100 & \\
\hline \multicolumn{6}{|c|}{ Idade gestacional (semanas) } \\
\hline 10 ou menos & 30 & 48,4 & 11 & 52,4 & 0,874 \\
\hline 11 a 12 & 15 & 24,2 & 6 & 28,6 & \\
\hline 13 a 16 & 14 & 22,6 & 3 & 14,3 & \\
\hline 17 e mais & 3 & 4,8 & 1 & 4,7 & \\
\hline Total & 62 & 100 & 21 & 100 & \\
\hline \multicolumn{6}{|c|}{ Hemorragia grave à admissão } \\
\hline Sim & 10 & 16,1 & 6 & 28,6 & 0,212 \\
\hline Não & 52 & 83,9 & 15 & 71,4 & \\
\hline Total & 62 & 100 & 21 & 100 & \\
\hline \multicolumn{6}{|c|}{ Sinais de infecção à admissão } \\
\hline Sim & 5 & 8,1 & 4 & 19,1 & 0,162 \\
\hline Não & 57 & 91,9 & 17 & 80,9 & \\
\hline Total & 62 & 100 & 21 & 100 & \\
\hline \multicolumn{6}{|c|}{ Tratamento com antibiótico } \\
\hline Sim & 2 & 3,2 & 4 & 19,1 & 0,016 \\
\hline Não & 60 & 96,8 & 17 & 80,9 & \\
\hline Total & 62 & 100 & 21 & 100 & \\
\hline
\end{tabular}

* Onze mulheres do grupo abortamento espontâneo não responderam se desejaram a gestação, pois não sabiam que estavam grávidas.

* Eleven women in the spontaneous abortion group did not answer if they wished their pregnancy, because they did not know that they were pregnant.

do abortamento espontâneo pensaram em abortar. Das mulheres que interromperam a gravidez, 47,6\% (n=10) delas foram aconselhadas a abortar e um pequeno percentual do outro grupo recebeu o mesmo conselho $(16,1 \%, n=10)$, diferenças estas estatisticamente significantes $(\mathrm{p}=0,004)$.

$\mathrm{O}$ método de escolha da maior parte das mulheres para a prática do abortamento foi o Misoprostol (Cytotec), demonstrado na Tabela 3. Quando somadas as porcentagens de uso da medicação sozinha e associada com outros métodos, o percentual de utilização entre as entrevistadas foi de $76,2 \%(n=16)$.

É interessante mencionar que, em nosso estudo, todas as mulheres que se inter- naram por complicações do abortamento foram submetidas à curetagem uterina.

Ao serem perguntadas sobre quem indicou o método para o abortamento, 71,4\% $(\mathrm{n}=15)$ das mulheres citaram amigos ou conhecidos, $14,3 \%(n=3)$ mencionaram familiares, $9,5 \%(n=2)$ disseram que foi o pai do concepto e $4,8 \%(n=1)$ afirmaram que recorreram a ele por conta própria.

Como se pode observar, na Tabela 3 , a opinião do pai do concepto no processo de tomada de decisão teve maior participação do que o ponto de vista da família em relação à prática do abortamento induzido, uma vez que nenhum tipo de participação da família foi referido por $61,90 \%$ $(n=13)$ das entrevistadas e, destas, $46,2 \%$ 
Tabela 2 - Distribuição dos grupos segundo decisão sobre o aborto, estratificada pelo tipo de abortamento. Table 2 - Group distribution according to decision to abort stratified by type of abortion.

\begin{tabular}{|c|c|c|c|c|c|}
\hline \multirow[b]{2}{*}{ Características } & \multicolumn{2}{|c|}{ Aborto Espontâneo } & \multicolumn{2}{|c|}{ Aborto Induzido } & \multirow[t]{2}{*}{$p$} \\
\hline & $\mathrm{N}$ & $\%$ & $\mathrm{~N}$ & $\%$ & \\
\hline \multicolumn{6}{|c|}{ Você pensou em abortar? } \\
\hline Sim & 11 & 17,7 & 21 & 100 & 0,000 \\
\hline Não & 51 & 82,3 & 0 & 0 & \\
\hline Total & 62 & 100 & 21 & 100 & \\
\hline \multicolumn{6}{|c|}{ Alguém a aconselhou a abortar? } \\
\hline $\operatorname{Sim}$ & 10 & 16,1 & 10 & 47,6 & 0,004 \\
\hline Não & 52 & 83,9 & 11 & 52,4 & \\
\hline Total & 62 & 100 & 21 & 100 & \\
\hline
\end{tabular}

Tabela 3 - Distribuição das mulheres que tiveram aborto induzido, segundo características do processo de tomada de decisão e método utilizado para o abortamento.

Table 3 - Distribution of women who had an induced abortion, according to the characteristics of the decision-making process and to the method used for abortion.

\begin{tabular}{|c|c|c|}
\hline Característicasz & $\mathrm{N}$ & $\%$ \\
\hline \multicolumn{3}{|l|}{ Participação do pai do concepto } \\
\hline Incentivou & 8 & 38,1 \\
\hline Apoiou & 5 & 23,8 \\
\hline Foi contra & 1 & 4,8 \\
\hline Nenhuma & 7 & 33,3 \\
\hline Total & 21 & 100 \\
\hline \multicolumn{3}{|l|}{ Participação da família } \\
\hline Incentivou & 2 & 9,5 \\
\hline Apoiou & 1 & 4,8 \\
\hline Foi contra & 5 & 23,8 \\
\hline Nenhuma & 13 & 61,9 \\
\hline Total & 21 & 100 \\
\hline \multicolumn{3}{|c|}{ Com quem conversou para decidir se abortava ou não } \\
\hline Amiga & 6 & 28,7 \\
\hline Pai da criança & 4 & 19,0 \\
\hline Mãe & 2 & 9,5 \\
\hline Tia/prima & 2 & 9,5 \\
\hline Ninguém & 7 & 33,3 \\
\hline Total & 21 & 100 \\
\hline \multicolumn{3}{|l|}{ Motivo } \\
\hline Falta de condições financeiras & 10 & 29,4 \\
\hline Falta de apoio do pai do concepto & 7 & 20,6 \\
\hline Relação instável & 6 & 17,7 \\
\hline Considera número atual de filhos suficiente & 4 & 11,8 \\
\hline Risco de perder o emprego/parar de estudar & 4 & 11,8 \\
\hline Medo da família & 2 & 5,8 \\
\hline Não querer a criança & 1 & 2,9 \\
\hline Total & $34^{*}$ & 100 \\
\hline \multicolumn{3}{|l|}{ Método utilizado } \\
\hline Cytotec associado a outros métodos & 1 & 4,8 \\
\hline Cytotec somente & 15 & 71,4 \\
\hline Outros métodos (Chás, drogas, etc) & 5 & 23,8 \\
\hline Total & 21 & 100,0 \\
\hline
\end{tabular}

* Resposta com mais de uma opção de escolha.

* The answer is multiple choice. 
(n=6) disseram não ter contado à família que estavam grávidas. Em contrapartida, $38,1 \%(\mathrm{n}=8)$ dos homens incentivaram e $23,8 \%(n=5)$ deles apoiaram, perfazendo um total de $61,9 \%(n=13)$, e apenas $33,3 \%$ $(n=7)$ não participaram do processo. Dos sete homens que não participaram do processo, quatro (19,0\%) deles nem ficaram sabendo da existência da gestação.

Em relação ao motivo que levou à indução do abortamento, dez mulheres referiram falta de condições financeiras, sete falta de apoio do pai do concepto, seis indicaram o fato de ter uma relação conjugal instável, quatro relacionaram a limitação da prole, considerando o número de filhos atual suficiente, quatro tiveram medo de perder o emprego e/ou ter que parar de estudar, duas citaram medo da família e apenas uma simplesmente justificou não querer a criança.

As mulheres que pensaram em abortar e realmente efetivaram o ato conversaram para decidir se abortavam ou não com: uma amiga $(28,7 \%, n=6)$, o pai do concepto $(19 \%, n=4)$, a mãe $(9,5 \%, n=2)$, a tia/prima $(9,5 \%, n=2)$ e com ninguém $(33,3 \%, n=7)$.

Em relação à opinião sobre o abortamento provocado, apenas uma mulher do grupo do abortamento induzido referiu ser a favor do aborto e não acreditar ter realizado um ato errado.

\section{Discussão}

Alguns fatores podem ter limitado os resultados encontrados, como o fato de ser possível que, na população estudada, alguns abortos classificados como espontâneos tenham sido provocados, uma vez que algumas mulheres podem ter ocultado a real classificação do abortamento e o fato de não ser conhecida a proporção de todos os abortos provocados na Serra que requerem hospitalização, limitações estas que têm o poder de subestimar as associações encontradas.

O percentual de abortamentos induzidos encontrados em nossa pesquisa $(25,3 \%)$ representa a proporção de abortos certa- mente induzidos, segundo classificação da $\mathrm{OMS}^{13}$ e foi semelhante aos achados em outros estudos ${ }^{14-16}$.

A maior parte das mulheres dos dois grupos não planejou a gestação. Como esperado, todas as mulheres do grupo do abortamento induzido referiram não tê-la planejado. Souza et al. ${ }^{14}$, ao entrevistarem mulheres internadas por complicações do abortamento no Instituto Materno-Infantil de Pernambuco, concluíram que mais de $60 \%$ das mulheres não queriam engravidar.

$\mathrm{O}$ fato de quatro entrevistadas pertencentes ao grupo do abortamento induzido terem afirmado ter desejado a gestação, apesar de não a terem levado até o fim, merece ser ressaltado, indicando que a escolha não está relacionada simplesmente ao fato de ser ou não ser mãe, e sim ao contexto em que a maternidade se dá, quer por incentivo ou apoio do parceiro em relação à decisão pelo abortamento ou até mesmo por sua não participação no processo de tomada de decisão, deixando clara o não planejamento da gravidez por parte do casal. Hardy e colaboradores ${ }^{17}$ discutem que embora a tendência atual seja instituir igualdade entre homens e mulheres, quando se pensa em regulação da fecundidade e saúde reprodutiva e sexual, praticamente não se fala sobre a participação masculina. E Duarte e colaboradores $^{18}$ completam que também não se fala da participação do homem quando o assunto é a interrupção da gestação.

Além da omissão masculina, alguns autores ${ }^{19,20}$ defendem que a escassez de informação sobre planejamento familiar e a falta de acesso a métodos contraceptivos resultam na propagação de gestações não desejadas e, conseqüentemente, no aumento de abortamentos em condições de risco.

A idade gestacional predominante ter sido de dez semanas e menos pode ter contribuído para o baixo índice de complicações apresentadas. Resultado semelhante foi encontrado por outros autores ${ }^{16,21}$.

Um maior percentual de mulheres do grupo do abortamento induzido apresentou hemorragia grave e sinais de infecção 
na admissão hospitalar, quando comparadas com o grupo do abortamento espontâneo. Esses dados apontados por outros estudos $^{13,16,22,23}$ deixam claro que o risco de complicações e de prejuízo para a saúde da mulher com posteriores seqüelas é maior em abortamentos realizados em condições inadequadas. Também é importante ressaltar o impacto econômico dessas complicações para os serviços públicos de saúde, gerado pelo gasto com tempo de internação, medicamentos e transfusões sanguíneas.

O método de escolha da maior parte das mulheres para a prática do abortamento foi o Cytotec. Percentual semelhante foi encontrado em outros estudos ${ }^{13,16,23-25}$. No estudo de Bailey et al. ${ }^{15}$, a introdução de corpos estranhos no útero foi o método mais citado.

Sabe-se que o uso do cytotec tem diminuído a porcentagem de mulheres que sofrem por complicações do abortamento, uma vez que o aumento da utilização desse medicamento faz com que métodos relacionados com altos índices de infecção, como introdução de corpos estranhos e utilização de outras técnicas invasivas para a indução do abortamento, sejam utilizados em menor freqüência. Osis e colaboradores ${ }^{26}$ acreditam que um elemento facilitador na decisão de abortar de algumas mulheres é o fato de o método utilizado, na maioria das vezes, ser administrado via oral.

Em nosso estudo, todas as mulheres que se internaram por complicações do abortamento foram submetidas à curetagem uterina, dado este que está de acordo com o estudo de Fonseca e colaboradores ${ }^{27}$.

Ao serem perguntadas sobre quem indicou o método para o abortamento, grande parte das entrevistadas referiu as amigas como a principal fonte de informação, o que corrobora com os achados de outros estudos $^{16,28}$. A opinião do pai do concepto no processo de tomada de decisão teve maior participação do que o ponto de vista da família em relação à prática do abortamento induzido. Outros estudos, no entanto, demonstram que a gestação não foi comunicada à maior parte dos parcei- $\operatorname{ros}^{13,28}$. Costa e colaboradores ${ }^{29}$ defendem que, enquanto amigos e parceiros incentivam projetos de vida de não aceitação de uma gestação indesejada, a família tende a valorizar o papel da mãe e a incentivar a manutenção da gravidez.

Em relação ao motivo que levou à indução do abortamento, a falta de condições financeiras foi o fator mais referido pelas mulheres entrevistadas seguido pela falta de apoio do pai do concepto, resultado este percebido em outros estudos ${ }^{13,14,24}$.

É importante ressaltar que os motivos que levam a mulher a praticar o abortamento na maioria das vezes estão interrelacionados, tendo quase sempre o envolvimento de mais de um fator, de tal modo que um influencia o outro em um processo dinâmico e complexo ${ }^{13}$.

$\mathrm{Na}$ maioria das vezes, a sociedade encara a decisão pela interrupção da gestação como uma decisão egoísta da mulher, desafiando códigos legais e morais ${ }^{29}$; entretanto, sabe-se que o abortamento leva a grande sofrimento físico e emocional. Osis e colaboradores ${ }^{26}$, ao estudarem a razão que levou mulheres a desistirem de abortar, destacam que o medo de serem presas não foi citado; os motivos citados foram relacionados às conseqüências de âmbito físico, psicológico e moral. Sabese que as complicações psicológicas podem levar a dispareunia, rejeição ao coito e ao uso de contraceptivos, como já foi demonstrado em outros estudos ${ }^{24,30}$, estando associadas ao sentimento de culpa, sentimento este que é retratado na fala de algumas de nossas entrevistadas em relação à opinião sobre o abortamento induzido: "É um grande pecado, eu achava que nunca faria isso, mas me vi obrigada a fazer"; "Acho errado, mas foi a única solução"; "Acho que não deveria acontecer, estou arrependida”.

\section{Considerações finais}

Ao se pensar na omissão do pai do concepto diante de uma gestação inesperada e na falta de participação da família 
no processo de tomada de decisão, analisa-se que, no presente estudo, esses dois fatores concorreram para consolidar a decisão pelo abortamento induzido. Deve-se refletir sobre o modelo predominante de masculinidade, no qual os homens se eximem de sua participação, tanto na prevenção da gravidez indesejada quanto na prática da paternidade responsável, e sobre a moral dominante que cultua o casamento como única possibilidade de vivência da sexualidade e da reprodução para as mulheres.

Assim, os motivos que levam as mulheres a abortarem são os reflexos não apenas de seus valores, mas, fundamentalmente, das normas sociais da cultura em que vivem. Destacam-se como causas as condições financeiras e a instabilidade marital, que revelam a ausência de estrutura familiar e o abandono da mulher, que, em alguns casos, mãe solteira, não se sente preparada psicologicamente, e muitas vezes financeiramente, para assumir um filho e vê a interrupção da gravidez como a melhor decisão a ser tomada.

Pelo exposto, acredita-se que o foco principal do problema do abortamento induzido é a gravidez indesejada, sendo necessário, portanto, aumentar as possibilidades de se planejar a gestação, valorizando o Planejamento Familiar. Este deve ser pautado em outros conceitos, não o de simplesmente reproduzir informações, e sim criar de espaços de diálogo para que não apenas as mulheres sejam protagonistas, mas que, principalmente, os homens possam ser envolvidos neste processo e se responsabilizem, participando das decisões pelo método contraceptivo e dividindo com as mulheres o direito a uma vida reprodutiva que seja guiada pela escolha consciente e pelo desejo de cuidar de uma nova vida.

\section{Referências}

1. Organização Pan-americana de Saúde (OPAS). Mortalidade materna-debate [S.I.: s.n.]; 2004. Disponível em: ww.opas.org.br/mostrant.cfm?codigodest=232. Acessado em 12 dezembro de 2005.

2. Organização Pan-americana de Saúde (OPAS). Profissionais capacitados para combater mortalidade materna [S.I.: s.n.]; 2005. Disponível em: http://66.102.7.104/custom?q=cache: ftARtegEljkJ:www.opas.org.br/rh/noticia_det.cfm\% 3Fid_noticia\%3D224+ mortalidade+materna\&hl=ptBR\&ie=UTF-8). Acessado em 12 dezembro de 2005.

3. Van Look PFA; Cottingham JC. Unsafe abortion: an avoidable tragedy. Best Pract Res Clin Obstet Gynaecol 2002; 16(12): 205-20.

4. Brasil. Código penal. São Paulo: Saraiva; 1999.

5. Costa SH. Aborto provocado: dimensão do problema e a transformação da prática. In: GIFFIN, Karen; COSTA, Sarah Hawker (Org.). Questões da saúde reprodutiva. Rio de Janeiro: Editora Fiocruz; 1999. p. 163- 84.

6. Galli, B. Aborto e Direitos Humanos: O impacto da criminalização do aborto na saúde e na vida das mulheres no Brasil [S.I.: s.n.], 2007. Disponível em: http:/ /www.ipas. org.br/arquivos/aborto_e_DH_BG07.doc. Acessado em 22 de setembro de 2007.

7. Organização Mundial da Saúde (OMS). International Women's Health Coalition. Abortamento Seguro: orientação técnica e política para os sistemas da saúde.
Campinas, SP: Cemicamp; 2004.

8. Monteiro MFG, Adesse L. Estimativas de aborto induzido no Brasil e Grandes Regiões (1992-2005). Rev Saúde Sex Reprod [periódico na Internet]. nov 2006 [citado $2007 \mathrm{Fev}$ 10 ]; (26): [cerca de 10p.]. Disponível em: http:/ / www.ipas.org.br/arquivos/ml2006.pdf. Acessado em 22 de setembro de 2007.

9. Brasil. Ministério da Saúde. Secretaria de Políticas de Saúde. Área Técnica de Saúde da Mulher. Parto, aborto e puerpério: assistência humanização à saúde. Brasília: Ministério da Saúde; 2003.

10. Espírito Santo. Prefeitura Municipal da Serra. Secretaria Municipal de Saúde. Sistema de Informação de Mortalidade. Sistema de Informação de Nascidos Vivos. Mortalidade materna. Serra; 2004.

11. Espírito Santo. Prefeitura Municipal da Serra. Secretaria Municipal de Saúde. Relatório de Autorizações de Internação Hospitalar. Serra; 2004.

12. World health organization (WHO). Protocol for hospital based descriptive studies of mortality, morbidity related to induced abortion. Geneva: WHO taste force on safety and efficacy of fertility regulating methods; 1987.

13. Lucena RCB. Características de mulheres internadas por aborto em uma maternidade pública em Recife - PE: dimensão do problema e sua relação com a prática contraceptiva. [dissertação de mestrado] Recife: Fundação Oswaldo Cruz. Centro de Pesquisa Aggeu 
Magalhães. Núcleo de Estudos em Saúde Coletiva; 2000.

14. Souza AI, Aquino MMA, Cecatti JG, Silva JLP. Epidemiologia do abortamento na adolescência. Rev Bras Ginecol Obstet 1999; 21(3): 161-5.

15. Bailey PE, Llano Saavedral L, Kushner L, Welsh M, Janowitz B. Estudio hospitalario del aborto ilegal em Bolívia. Bol Sanit Panam 1988; 104(2): 144-59.

16. Souza AI, Cecatti JG, Ferreira LOC, Santos LCet. Hospitalização por abortamento no centro de atenção à mulher (CAM) - IMIP - Recife. J Bras Ginecol 1996; 106(9): 355-61.

17. Hardy E, Costa RG, Rodrigues T, Morais T M. Características atuais associadas à história de aborto provocado. Rev Saúde Pública 1994; 28(1): pp ???

18. Duarte GA, Alvarenga AT, Osis M.J.M.D, Faúndes A, Hardy E. Perspectiva masculina acerca do aborto provocado Rev Saúde Pública 2002; 36(3): pp ???

19. Faúndes A, Duarte GA, Neto JA, Olivatto AE, Simonete RM. Conhecimento, opinião e conduta de ginecologistas e obstetras brasileiros sobre o aborto induzido. Rev Bras Ginecol Obstet 2004; 26(2): pp ??? Disponível em: www.bireme.br. Acessado em 14 de janeiro de 2005.

20. Costa CFF, Costa HLFF, Machado KM, Melo MPV, Rocha YKW, Costa LOBF. Aspectos epidemiológicos comparativos entre abortamento provocado e espontâneo. Rev Bras Ginecol Obstet 1996; 18(2): 197-84.

21. Schor N. Investigação sobre a ocorrência de aborto em pacientes de hospital de centro urbano do Estado de São Paulo, Brasil. Rev Saúde Pública 1990; 24(2): 144-51.

22. Fonseca AD, Maas CG, Lipinsk JM, Soares SS. Abortamento: nossa realidade. Rev Enferm UERJ 1997;
5(1):317-24.

23. Fonseca W, Misago C, Correia LL, Parente JMA, Oliveira FC. Determinantes do aborto provocado entre mulheres admitidas em hospitais em localidade da Região Nordeste do país. Rev Saúde Pública 1996; 30(1): pp ???

24. Parente ACBV, Lima AKA, Silva FCB, Carvalho MLB, Parente JV. Etiologia do aborto criminoso em Terezina. RASPP - Ver Assoc Saúde Pública Piauí 1998; 1(2): 137-45.

25. Moretti E, Rovani IM, Vargas M. Perfil das pacientes em abortamento em setor de emergência. Rev Med HSVP 1998; 10(23): 15-20.

26. Osis MJD, Hardy EE, Faúndes A, Rodrigues T. Dificuldades para obter informações da população de mulheres sobre aborto ilegal. Rev Saúde Pública 1996; 30(5): pp ???

27. Fonseca W, Misago C, Freitas P, Santos E, Fernandes L, Correia L. Características sócio-demográficas, reprodutivas e médicas de mulheres admitidas por aborto em hospital da Região Sul do Brasil. Cad Saúde Pública 1998; 14(2): pp ??? Disponível em: www.bireme.br. Acessado em 14 de janeiro de 2005.

28. Osava RH, Amorim MHC, Castro DS, Marques MS. Perfil reprodutivo e padrões de consumo do misoprostol (cytotec) como droga abortiva em usuárias de baixa renda. Rev Enferm UERJ 1997; 5(1): 325-30.

29. Costa RG, Hardy E, Osis MJD, Faúndes A. A decisão de abortar: processos e sentimentos envolvidos. Cad Saúde Pública 1995; 11(1): 97-105.

30. Bruno ZV. Abortamento: aspectos epidemiológicos. Femina: Rio de Janeiro; 1993. p. 912-16.

Recebido em: 19/03/07

Versão final reapresentada em: 15/10/07

Aprovado em: 09/11/07 